\title{
Absolute parameters for binary systems
}

\section{The active binary $\mathrm{BH}$ Virginis}

\author{
R. Clement ${ }^{1}$, V. Reglero ${ }^{1}$, M. Garcia ${ }^{2}$, J. Fabregat ${ }^{1}$, and J. Suso ${ }^{1}$ \\ 1 Departamento de Astronomía y Astrofísica, Universidad de Valencia, Dr Moliner 50, E-46100, Burjassot, Valencia, Spain \\ e-mail: clement@deneb.matapl.uv.es \\ 2 Departamento de Física Aplicada, E.U.I. Industrial, Universidad Politécnica de Madrid, Ronda de Valencia 3, E-28012 Madrid, \\ Spain
}

Received September 12; accepted December 6, 1996

\begin{abstract}
New high-quality light curves of the late-type binary system BH Vir have been obtained during a 6 year photometric $u v b y$ and $\mathrm{H} \beta$ monitoring program of low mass eclipsing binaries (Clement et al. 1997), hereafter Papers I and II.

This paper presents detailed analysis of this binary based on the four light curves obtained within our program. The activity wave superimposed on the eclipsemodulated light curves has been adjusted and removed by using a new iterative application of the standard EBOP code together with truncated Fourier Series fittings. Combining the recent radial velocity curves (Popper 1995) with the geometrical elements deduced from the "clean" photometric light curves, the absolute parameters of the system are calculated. BH Vir appears as a binary composed by two main sequence G0 and G2 stars with solar chemical composition lying on the same isochrone $(\log (\tau)=9.8 \pm 0.2$ years $)$.

The accuracy obtained on the absolute parameters determination allows us to include $\mathrm{BH}$ Vir on the select group of late-type stars to be used on the estimation of the Mass-Luminosity-Relationship.
\end{abstract}

Key words: stars: binaries: eclipsing - stars: late-type - stars: activity - stars: fundamental parameters - stars: individual: BH Vir — stars: evolution

\section{Introduction}

More accurate observational determinations of masses, radii, temperatures and abundances are needed in order to improve the mass-luminosity relation at the end of the

Send offprint requests to: $\mathrm{R}$. Clement
Main Sequence. Those physical quantities are poorly defined in that mass range where only a few stars have astrophysical parameters calculated with the required accuracy. New good-quality light curves of some late-type binary systems have been obtained during a 6 year photometric observational $u v b y$ and $\mathrm{H} \beta$ monitoring program of low mass eclipsing binaries (Paper I and Paper II). In this framework, particular attention was paid to $\mathrm{BH}$ Vir, for which new radial velocity curve has been recently obtained (Popper 1995).

Photometric studies of the interesting eclipsing binary BH Vir have been carried out by several authors (Kitamura et al. 1957; Koch 1967; Sadik 1978; Botsula 1978; Hoffmann 1982; Scaltriti et al. 1985). More recently in a detailed analysis of the available light curves using the Wilson-Devinney code, Zhai et al. (1990) derived the relevant astrophysical parameters of both components. Vincent (1993), using surface imaging techniques includes $\mathrm{BH}$ Vir in the list of binary systems undergoing total eclipse.

The analysis of the photometric light curves for $\mathrm{BH}$ Vir is a difficult task mainly due to the large and very fast amplitude variations outside eclipses. Koch (1967), reported changes in the depth of the primary eclipse of a tenth of a magnitude, in only few days. The most generally accepted hypothesis to explain this light curve modulation outside eclipse is that the coldest star (or sometimes both) displays surface activity phenomena similar to the Sun, but of greater magnitude. The large body of existing data demonstrates that solar activity is a general phenomenon in stars of spectral type later than A. Rotation and convection are the basic ingredients (Hartmann \& Noyes 1987).

Despite of showing intrinsic variability, BH Vir has been included in our program due to the lack of late-type eclipsing binaries without photometric variations outside eclipse. Relevant photometric elements can be extracted for such systems too, once the light curves have been 
cleaned of the activity waves attributed to the presence of spots in the stellar surface. We have implemented a new method to clean the light curves of the modulation due to activity. This method combines the determination of the activity wave with the search for the binary solution in an iterative process.

In this paper we present the detail analysis of BH Vir, based on the good-quality uvby and $\mathrm{H} \beta$ light curves which has been fully covered at least three times in four different epochs (Paper II).

\section{The observations}

Initial light curves were obtained in March 1990 and January 1992 at The Calar Alto Observatory (Almeria, Spain). The telescope used was the $1.5 \mathrm{~m}$ reflector equipped with a multipurpose one-channel photoelectric photometer or, with a four-channel $u v b y-\mathrm{H} \beta$ spectrograph photometer, both using narrow and wide $\mathrm{H} \beta$ filters. The last two photometric campaigns were carried out in April 1993 and May 1994 at The European Southern Observatory (La Silla, Chile) using the $0.5 \mathrm{~m}$ Danish telescope equipped with a uvby- $\mathrm{H} \beta$ four-channel photometer, identical to the one used at Calar Alto, using Strömgren uvby filters as well as Crawford's narrow and wide $\mathrm{H} \beta$ filters. Standardised values for BH Vir and its comparison stars have been given in Paper I, as well as an indication of the accuracy of the photometry. In Paper II the new good-quality light curves for BH Vir have been given.

\section{Reddening and radiative parameters of the comparison stars}

Before going into the analysis of the associated wave and the light curve of $\mathrm{BH}$ Vir itself, we have estimated the local interstellar extinction using the comparison stars.

HD 121935, $\left(m_{v}=9.67, F 4 V\right)$ and HD $139643\left(m_{v}=\right.$ $9.44, G 0 \mathrm{~V})$, were used as comparison stars. We checked their constancy every night. We found a RMS dispersion for the 564 differential magnitudes measured of 0.012 , $0.010,0.015,0.021$, and 0.017 for $V,(b-y), m_{1}, c_{1}$ and $\beta$ respectively. The standard value obtained for the comparison star, HD 121935, was $9.680 \pm 0.013,0.260 \pm 0.009$, $0.169 \pm 0.014,0.488 \pm 0.020$, and $2.670 \pm 0.016$, for $V$, $(b-y), m_{1}, c_{1}$ and $\beta$ respectively.

To estimate the local reddening we have followed a procedure similar to that described in Reglero et al. (1990). We obtained $E(b-y)=0.031$ and $E(b-y)=0.021$ for HD 121935 and HD 139643 respectively, with a mean value of $E(b-y)=0.026$. This value agrees well with the one calculated previously in Clement et al. (1993). The small apparent angular distance between the comparisons and BH Vir and their location at 208 and $106 \mathrm{pc}$ behind and in front of $\mathrm{BH}$ Vir, suggests the same interstellar extinction for BH Vir itself. We have adopted the mean value as the reddening for $\mathrm{BH}$ Vir.
After correcting magnitudes and indices for reddening, radiative parameters have been derived for both stars, using the well know standard calibration procedure for $\mathrm{F}$ type stars given by Crawford (1975). This method assumes the $\mathrm{H} \beta$ index as a reddening free independent parameter. Effective temperatures, $T_{\text {eff }}$, and visual surface brightness, $F_{\mathrm{v}}$, were derived from the semiempirical calibrations of Saxner \& Hammarback (1985) and Moon (1985) respectively. Both comparisons appear to be main sequence stars with solar metal abundances and located at 208 and $106 \mathrm{pc}$ respectively. Their spectral types can be estimated, from the present photometric calibrations, as F4 and G0 stars respectively. Their location in the HR and photometric $m_{1}-(b-y)$ and $c_{1}-(b-y)$ diagrams indicate a luminosity class $\mathrm{V}$ for both. The derived physical quantities for the comparisons of BH Vir are listed in Table 1. The errors on the table are the ones indicated for the calibrations, and for the distance the error is obtained through error propagation in the formula.

Table 1. Comparison stars, radiative parameters

\begin{tabular}{lrrrrrl}
\hline name & $M_{v}$ & $F_{\mathrm{v}}$ & {$[\mathrm{Fe} / \mathrm{H}]$} & $T_{\text {eff }}$ & $d$ & Sp \\
\hline & & & & & & \\
HD 121935 & 3.1 & 3.83 & 0.1 & 6590 & 208 & F4 V \\
& 3 & 3 & 2 & 60 & 27 & \\
HD 139643 & 4.3 & 3.76 & -0.1 & 5914 & 106 & G0 V \\
& 3 & 3 & 2 & 60 & 14 & \\
\hline
\end{tabular}

\section{Discussion}

\subsection{Activity of BH Vir}

As we said in the introduction, it is generally accepted that many late-type fast rotator stars show surface activity phenomena. As a consequence of the quasisynchronisation of the rotation and orbital periods of many sufficiently evolved late-type binary systems, a variable fraction of the active zones or spots of a star within the system (or both) can be seen throughout each complete period, producing a magnitude variation outside eclipse. Assuming a solar standard model of activity, the position of the spots at different latitudes can give rise to migrations of the associated wave, giving the spots a lower velocity (spots at high latitudes) or higher velocity (spots close to the equator) relative to the average rotation velocity. The latter is the velocity synchronised with the period of the binary system, thus the migration of the spots adds complexity to the task of analysing the light curves. Simultaneously, other specific effects are produced due to the proximity of the two stars, such as deformations due to gravitational interaction, and mutual reflection, that also generate variations in the light curves between minima. 
A preliminary inspection of the $\mathrm{BH}$ Vir light curves clearly shows a distortion outside the eclipses, called the associated wave, see Fig. 1. Amplitude variations up to 0.1 magnitudes in the $y$ filter are clearly visible at phases 0.1 an 0.2 .

So, before going into the analysis of the light curve it is necessary to clean the associated wave.

Different systems to clean the activity have been used and reported in the literature. Ludington (1978) was the first to use truncated Fourier Series (four terms) to fit the observed points outside eclipse and later to compute the geometrical solution of the binary.

The main problem with this cleaning process is that binary effects between components are also included in the terms of the Fourier Series and the rectified light curve becomes flat between eclipses losing relevant information. Proximity effects are clearly included in the second order terms of the Fourier Series.

In order to avoid this problem, a new approach is presented in this paper. We have used an iterative process that:

- adjusts a preliminary wave by Fourier Series to the points outside eclipses (zero-order wave)

- computes a solution for the system, from a zero-order solution using the rectified light curve (first order solution)

- adjusts a new associated wave (first-order wave) by fitting a Fourier Serie to the residuals (observed-first order solution) assuming that these residuals are mainly due to activity.

After this preliminary analysis the computed wave is subtracted from the initial light curve and the clean light curve is used to compute a new binary solution. The new solution is then used to recalculate a new wave, by using the residuals. The iterative process was stopped when the wave and geometric solution for the binary system became stable within the uncertainty of the binary solution. The successive binary solutions are computed using the well known EBOP code written by Etzel (1981). This program is adequate to synthesise detached binary system with little effects due to proximity. The radii relative to orbit of the components of $\mathrm{BH}$ Vir, 0.26 and 0.24 are small enough for EBOP to be adequate.

To estimate the associated waves from the data of April 1993 and May 1994 in which the light curves taken were well covered, we have used the above referred to iterative process. After 6 iterations the process converged (the differences in the coefficients for the consecutive waves were less than 0.001 , and the binary solution variations were less than the accuracy attained).

A main feature is the decrement on the wave amplitudes. It was found that the "real" amplitudes of the associated waves for these two campaigns are only $30 \%$ and $50 \%$, in the $y$ filter, of those calculated in the first approximation, measured as the integral of the wave over a whole orbital period. Clearly, in the zero-order solution there is a systematic overestimation of the activity wave by inclusion on the apparent waves of binary effects. Depending on the spot location over the stellar surface, overestimation or underestimation of the activity wave are expected in the zero-order approach. These results for BH Vir activity indicate the necessity to apply a more sophisticated deconvolution process than the zero-order approach.

The waves show the high level of variability of BH Vir with a mean variation amplitude of 0.07 magnitudes in the $y$ filter, measured as the difference between the waves at the point of maximum light. For the other filters, the wave pattern is very much the same. The waves calculated for March 1990 and January 1992 are somehow uncertain because they are based only in 19 and 23 normal points outside eclipses respectively. Due to the small number of points in these two epochs, we could not apply the iterative process to estimate the contribution of the activity. We just estimated the wave adjusting a truncated Fourier series to the residuals obtained with the best binary solution computed for the "clean" light curves of other two epochs, (1543 points). The coefficients found for the $u, v$, $b$ and $y$ waves respectively are:

\begin{tabular}{lrrrr} 
coeff. & March 1990 & January 1992 & April 1993 & May 1994 \\
\hline$A_{0}$ & -0.015729 & +0.007811 & +0.019489 & +0.020132 \\
$A_{1}$ & -0.035625 & -0.012013 & +0.005832 & +0.017463 \\
$A_{2}$ & -0.016264 & +0.023505 & -0.007779 & +0.000981 \\
$A_{3}$ & +0.008105 & -0.001278 & +0.004047 & +0.015410 \\
$A_{4}$ & +0.010965 & +0.011224 & -0.016751 & +0.004881 \\
& & & & \\
$A_{0}$ & +0.004730 & +0.010743 & +0.017115 & +0.014607 \\
$A_{1}$ & -0.044672 & -0.015328 & -0.000763 & +0.013777 \\
$A_{2}$ & +0.004087 & +0.024259 & -0.005253 & +0.003696 \\
$A_{3}$ & +0.005884 & -0.008475 & +0.001284 & +0.015283 \\
$A_{4}$ & +0.022943 & +0.009589 & -0.012563 & +0.003040 \\
& & & & \\
$A_{0}$ & +0.016240 & +0.018524 & +0.016169 & +0.012103 \\
$A_{1}$ & -0.033652 & -0.010165 & -0.003506 & +0.011396 \\
$A_{2}$ & -0.002286 & +0.025981 & -0.007304 & +0.004087 \\
$A_{3}$ & +0.002034 & -0.011804 & +0.000903 & +0.012935 \\
$A_{4}$ & +0.014351 & +0.007047 & -0.008284 & +0.003509 \\
& & & & \\
$A_{0}$ & +0.014442 & +0.014625 & +0.013222 & +0.013493 \\
$A_{1}$ & -0.033050 & -0.008445 & -0.004958 & +0.011500 \\
$A_{2}$ & +0.000443 & +0.020879 & -0.007114 & +0.002866 \\
$A_{3}$ & +0.000748 & -0.009356 & -0.001119 & +0.010735 \\
$A_{4}$ & +0.016629 & +0.007618 & -0.004876 & +0.005642 \\
& & & & \\
\hline
\end{tabular}

Figures 2, 3, 4, 5 show the estimated waves for each campaign in each filter. The waves corresponding to the first campaign are not reliable because the residual points used for the fitting are not homogeneously distributed, and they are not drawn.

The new method used to estimate the waves is physically correct and is clearly effective looking at the small dispersion of the points in the rectified light curve (Fig. 1), compared with the dispersion of points in the observed light curve (Fig. 7), specially outside eclipses, taking into account that they include points from four different campaigns along four years. To evaluate the goodness of the method we calculated normal points, binning the data in 


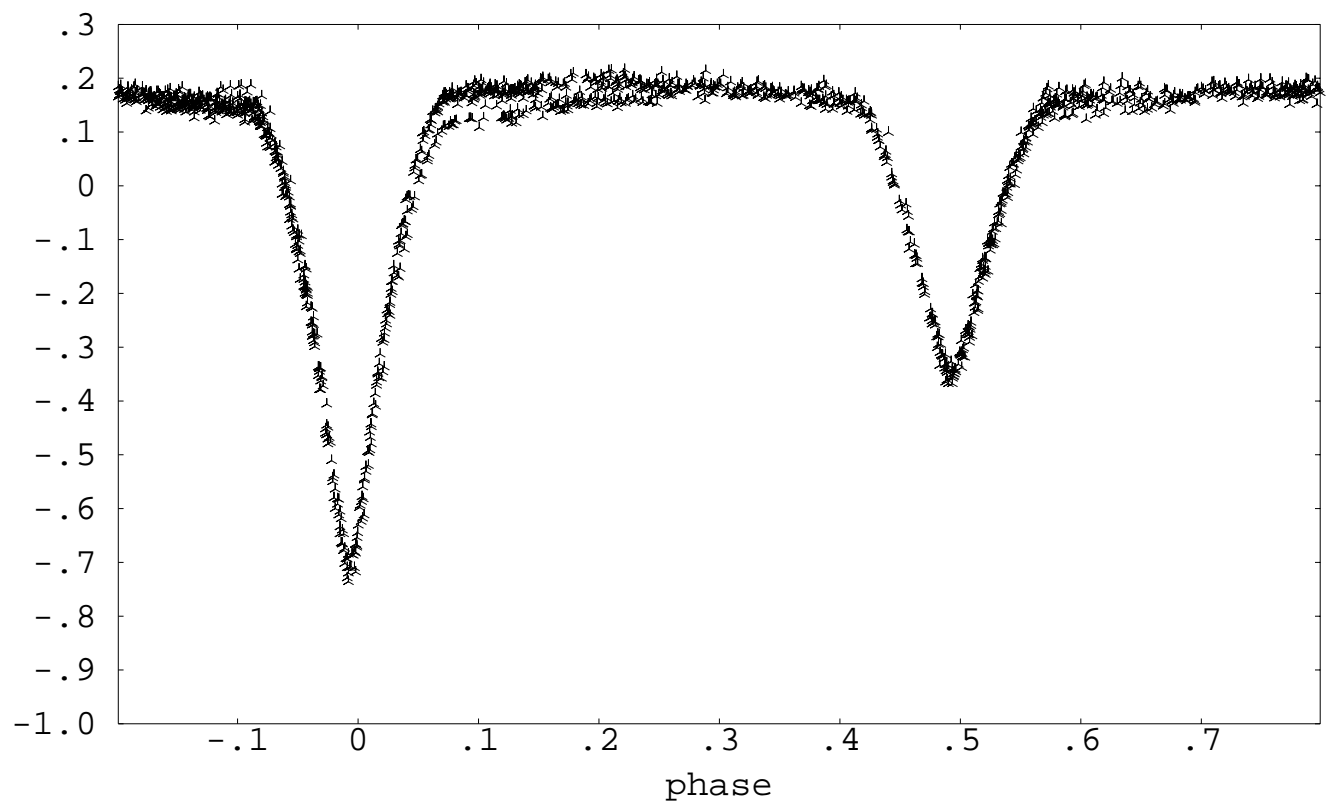

Fig. 1. Differential light curve BH Vir, $y$ filter

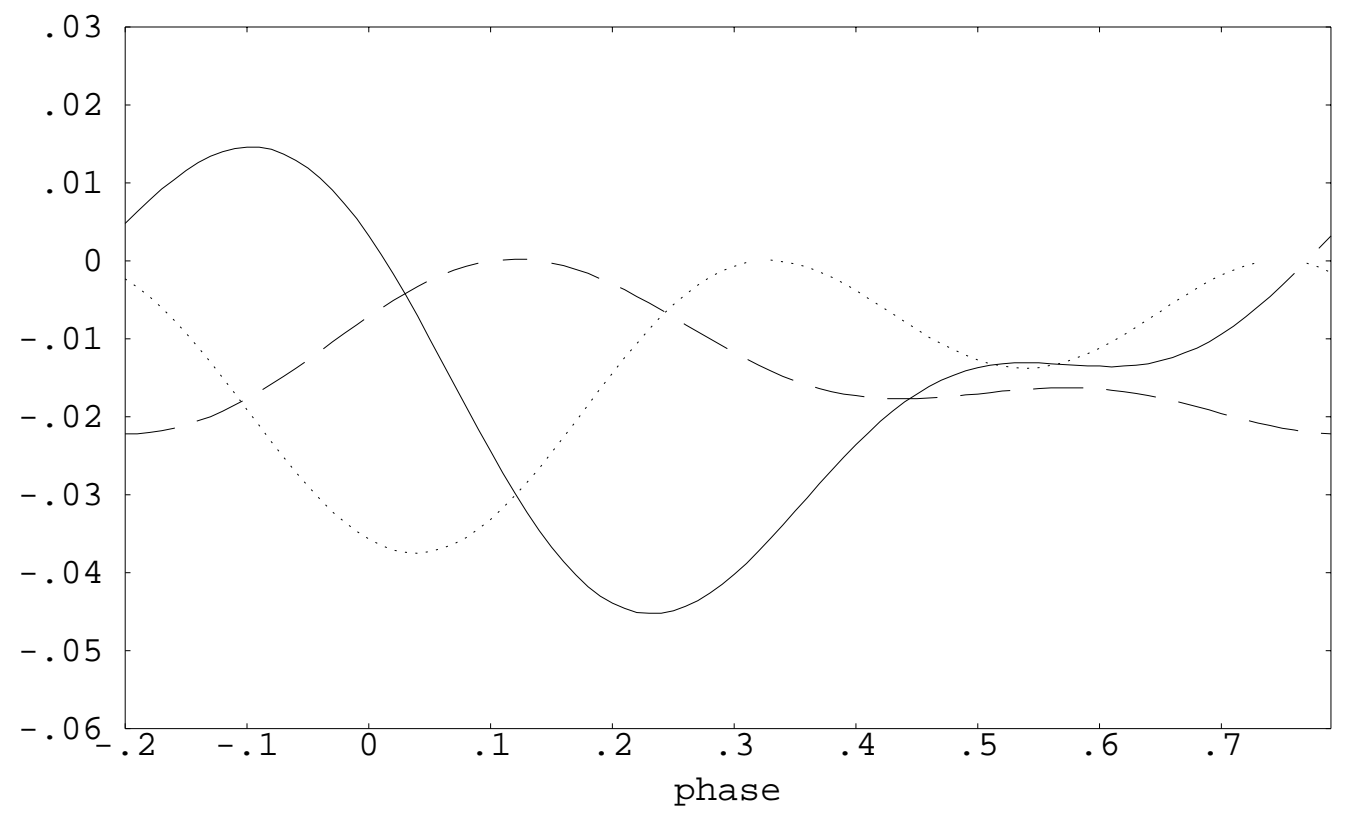

Fig. 2. Final waves Jan92, Apr93 and May94, y filter

intervals of 0.01 of phase, before and after "cleaning" in two different regions: outside eclipses and in different areas inside the eclipses. In the rectified light curves the RMS of all the normal points outside eclipses is equal or less than the accuracy of the photometry (0.009 in the $y$ filter) while in the non rectified ones is of the order 0.020 . Inside eclipses the scatter gets better. The dispersion at the bottom of the secondary is of the same order before and after cleaning. In the primary the dispersion of the 377 points at phases $[-0.08,0.08)$ improves a mean of 0.007 with a maximum at the right end of the primary $[0.07,0.08)$ where the 18 points binned go from RMS 0.033 to 0.015 in the $y$ filter. However the best global effect of the cleaning occurs from phase 0.06 to phase 0.23 and from phase 0.61 to 0.68 where the mean RMS change from $0.023,0.021$, $0.023,0.023$ to $0.014,0.010,0.010,0.008$ for the 661 points binned in $u, v, b$ and $y$ filters respectively. 


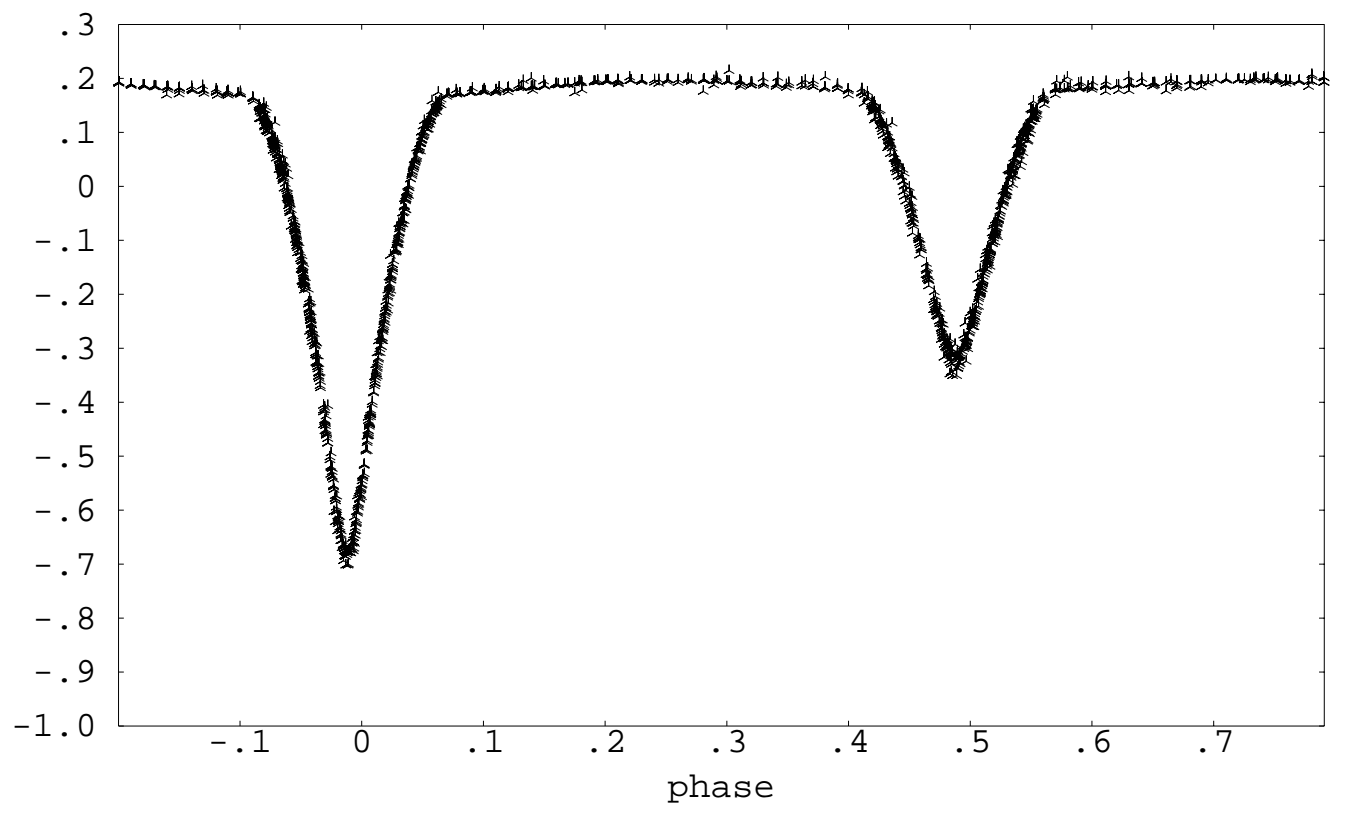

Fig. 7. BH Vir rectified light curve + EBOP solution. $y$ filter

eclipse appears to be total within the uncertainty of the luminosity errors, result that is in good agreement with Vincent (1993). In Table 5 we list the final EBOP solution. The geometrical results obtained for the components of BH Vir are consistent with those obtained by Zhai, although ours improve the accuracy of the radii of the components relative to the orbit, measured as the RMS of the values in the four filters. Zhai obtained $0.2619 \pm 0.0017$ and $0.2365 \pm 0.0019$, for $r_{\mathrm{p}}$ and $r_{\mathrm{s}}$, while we obtained $0.2634 \pm 0.0006$ and $0.2318 \pm 0.0002$.

Figure 7 shows the rectified light curve together with the EBOP best solution. The decrease in the $\langle(\mathrm{O}-\mathrm{C})\rangle$ (observed-calculated), and the disappearance of systematic variations in $(\mathrm{O}-\mathrm{C})$ in the final solution respect to the initial ones, in spite of merging points from four different campaigns of this active binary, also comfirms that the iterative method followed is efficient.

\subsection{Radiative parameters of $B H$ Vir}

In order to derived the astrophysical parameters of the individual components of $\mathrm{BH}$ Vir from the rectified light curves, after geometrical solution, we need to estimate the individual magnitudes and colour indices of each star instead of the integrated values. First of all we corrected magnitudes and indices of the local interstellar reddening estimation $E(b-y)=0.026$. We can use the photometric values during totality (the secondary eclipse), together with the $J_{\mathrm{p}}$ computed running EBOP, to get the individual values for each of the components. To derive the photometric values for the blue component we followed the usual technique given by Lacy (1977). In Table 2 magnitudes and indices are given outside eclipse (BH Vir) and during totality (blue component), together with the computed magnitudes and indices for the secondary star (red component).

Table 2. BH Vir. Rectified intrinsic magnitudes and indices

\begin{tabular}{lrrrrr}
\hline name & $y_{0}$ & $(b-y)_{0}$ & $m_{0}$ & $c_{0}$ & $\beta$ \\
\hline BH Vir & 9.504 & 0.369 & 0.185 & 0.312 & 2.592 \\
& 7 & 5 & 9 & 12 & 7 \\
BH Vir Hot & 10.019 & 0.348 & 0.184 & 0.333 & 2.627 \\
& 7 & 5 & 9 & 12 & 7 \\
BH Vir Cold & 10.560 & 0.403 & 0.190 & 0.275 & 2.572 \\
& 7 & 5 & 9 & 12 & 7 \\
\hline
\end{tabular}

The radiative parameters for the hot and cool components have been derived by using the well know standard calibration procedure for $\mathrm{F}$ type stars given by Crawford (1975), and extended by Olsen (1988), for early $\mathrm{G}$ type stars. Effective temperatures, $T_{\text {eff }}$, and visual surface brightness, $F_{\mathrm{v}}$, were computed from the semiempirical calibrations of Saxner \& Hammarback (1985), and Moon (1985), respectively. For the cool component we have used the standard photometric calibrations given by Olsen (1984), for late-type stars. Results are given in Table 3. Effective temperatures, visual flux brightness $F_{\mathrm{v}}$ and absolute magnitudes led us to classify the hot component as a G0 main sequence star. The cool component can be classified as a G2 main sequence star, both with solar composition. The distance, $135 \mathrm{pc}$, computed from the calculated absolute magnitude $M_{v}$, is slightly smaller than the one found previously (Clement et al. 1993), when 
we analysed the data for the three first campaigns, but is inside the indetermination of the $M_{v}$ value. As we can see the comparisons are located at a comparable distance.

Table 3. Radiative Parameters for BH Vir

\begin{tabular}{lrrrrrl}
\hline name & $M_{v}$ & $F_{\mathrm{v}}$ & {$[\mathrm{Fe} / \mathrm{H}]$} & $T_{\text {eff }}$ & $d$ & Sp \\
\hline BH Vir Hot & 4.37 & 3.77 & 0.1 & 6158 & 135 & G0 V \\
& 3 & 3 & 2 & 60 & 19 & \\
BH Vir Cold & 4.41 & 3.74 & -0.3 & 5714 & 170 & G2 V \\
& 3 & 3 & 2 & 60 & 22 & \\
\hline
\end{tabular}

\subsection{Mass, radius and evolutionary status}

Once the solution of the binary system was determined with sufficient precision - that is, $J_{\mathrm{s}}, r_{\mathrm{p}}, r_{\mathrm{s}}$ and $i$-, combining these values with the amplitudes $K_{1}$ and $K_{2}$, deduced from the radial velocity curve (Popper 1995), and with the values of the effective temperature and the flux of the primary star, $T_{\text {eff }}$ and $F_{\mathrm{v}}$ determined from the photometric calibration of the theoretical optimum light curve computed with EBOP, we calculated the absolute parameters: masses, radii, surface gravity, luminosity and bolometric magnitude. We used the astrophysical fundamental formula collected in Schimdt-Kaler et al. (1982). Table 6 list these values. The errors are obtained through the formula.

The masses calculated in this paper differ from the ones published by Koch (1967), Guiricin et al. (1984) and Zhai et al. (1990) because they are based in new values of $K_{1}$ and $K_{2}$ derived recently by Popper (1995) and new $i$ value.

The radii in this paper are also different from the calculated by other authors and more precise, Zhai gave $R_{\mathrm{p}}=1.166 \pm 0.036$ and $R_{\mathrm{s}}=1.048 \pm 0.034$ while we obtained $R_{\mathrm{p}}=1.250 \pm 0.007$ and $R_{\mathrm{s}}=1.114 \pm 0.006$. The fact that the photometric light curve has been clean with a more sophisticated method leads to different values of $A$. Zhai reported $A=4.37 \pm 0.11$ while we found $A=4.74 \pm 0.03$.

We used the recent evolutionary model of Claret \& Giménez (1992) to estimate the age of BH Vir and confirmed that both components lye on the same isochrone. The initial parameters for the model calculations were the masses given in Table 6 and solar metalicity. The temperatures, gravities and ages predicted with the model are: $\log \left(T_{\text {eff }}\right)=3.78 \pm 0.01, \log (g)=4.30 \pm 0.03$ and $\log (\tau)=9.7 \pm 0.2$ for the primary star and $\log \left(T_{\text {eff }}\right)=$ $3.761 \pm 0.008, \log (g)=4.35 \pm 0.03$ and $\log (\tau)=9.9 \pm 0.2$ for the secondary star, which gives an estimated age of $\log (\tau)=9.8 \pm 0.2$ for the system. The temperature and gravity calculated by this model agree with our calculations given in Table 6 . The evolutionary branch of this model can be seen in Fig. 6 .

\section{Conclusions}

We have presented an accurate determination of absolute parameters for the binary system BH Vir, based on new good-quality uvby $\beta$ light curves and new spectroscopic determination of mass ratio.

Before going into the analysis of the light curves we cleaned them of the contribution of the intrinsic activity, using a new iterative process that combines the wave determination with the binary solution search.

The effectiveness of this method, physically correct, is clearly seen looking at the reduction of the dispersion of the light curve outside eclipses which contains points from four different epochs of this highly variable binary system.

The geometric and photometric results agree well with the ones published by other authors but the precision attained in this work is one order of magnitude better. However the masses and radii derived in this paper are slightly different, since we used new and more accurate values for the amplitudes of the radial velocity curve, combined with the high accurate determination of the geometrical parameters from the photometric light curves. The secondary eclipse is total within the uncertainty of our analysis supporting the result of Vincent (1993).

So we can conclude that BH Vir is a binary composed by two main sequence stars of spectral type G0 and G2 that undergo total eclipse. The high level of activity which is variable with time has been removed from the light curve and then its absolute astrophysical parameters computed precisely.

This results allow us to include BH Vir on the select group of late-type binaries to be used on the computations of Mass-Luminosity Relationship (MLR).

Acknowledgements. The $1.5 \mathrm{~m}$ telescope at Calar Alto is operated by the Observatorio Astronómico Nacional. The $0.5 \mathrm{~m}$ telescope is operated by the Copenhagen Group. We want to express our gratitude to the observatories staff. This work has been supported by the Spanish Comisión Interministerial de Ciencia y Tecnología (PB90-0001-C02)

\section{References}

Binnendijk L., 1960, in: Properties of Double Stars. U. of Pennsylvania Press, Philadelphia, p. 288

Botsula R.A., 1978, in: Variable Star (Russian) 20, 577

Claret A., Giménez A., 1990, A\&A 230, 412

Claret A., Giménez A., 1992, A\&AS 96, 255

Clement R., Reglero V., García M., Fabregat J., Bravo A., Suso J., 1993, in: IAU Colloquium 137, "Inside the Stars", p. 386

Clement R., 1995, Doctoral Thesis, Valencia University

Clement R., García M., Reglero V., Clausen J.V., Bravo A., Suso J., Fabregat J., Torrejón J.M., Reig P., Marco A., 1997, A\&AS 123, 1 (Paper I)

Clement R., García M., Reglero V., Clausen J.V., Bravo A., Suso J., Fabregat J., 1997, A\&AS 123, 59 (Paper II)

Crawford D.L., 1975, AJ 80, 955

Etzel P.B., 1981, Masters Thesis. San Diego State University 
Table 4. BH Vir. Initial EBOP parameters(S0)

\begin{tabular}{|c|c|c|c|c|c|}
\hline parameters & number & $u$ & $v$ & $b$ & $y$ \\
\hline$J_{\mathrm{s}} / J_{\mathrm{p}}$ & $V-1$ & & & & 0.895 \\
\hline$r_{\mathrm{p}}$ & $V-2$ & 0.20 & & & \\
\hline$r_{\mathrm{s}} / r_{\mathrm{p}}$ & $V-3$ & 1.0 & & & \\
\hline prim. limb dark. coeff. & 4 & 0.85 & 0.82 & 0.76 & 0.67 \\
\hline second. limb dark. coeff. & 5 & 0.88 & 0.84 & 0.78 & 0.69 \\
\hline$i$ & $V-6$ & 88.0 & & & \\
\hline$e \cdot \cos (\omega)$ & 7 & 0 & & & \\
\hline$e \cdot \sin (\omega)$ & 8 & 0 & & & \\
\hline prim. gravita. dark. coeff. & 9 & 0.25 & & & \\
\hline second. gravita. dark. coeff. & 10 & 0.25 & & & \\
\hline prim. reflection (internal calculation) & 11 & 0 & & & \\
\hline second. reflection (internal calculation) & 12 & 0 & & & \\
\hline masses ratio & 13 & 0.967 & & & \\
\hline lead/lag ang & 14 & 0 & & & \\
\hline third light & 15 & 0 & & & \\
\hline out of phase & $V-16$ & & & & \\
\hline maximum light & $V-17$ & 0.008 & 0.054 & -0.073 & -0.170 \\
\hline integration ring & 18 & 5 & & & \\
\hline period & 19 & 0.81687099 & & & \\
\hline initial epoch- 2440000 & 20 & 3230.609 & & & \\
\hline
\end{tabular}

Table 5. BH Vir. EBOP final values

\begin{tabular}{|c|c|c|c|c|c|c|}
\hline parameters & number & $u$ & $v$ & $b$ & $y$ & $\sigma$ \\
\hline$J_{\mathrm{s}} / J_{\mathrm{p}}$ & $V-1$ & 0.59765 & 0.60404 & 0.65071 & 0.68763 & \\
\hline$\sigma$ & \pm & 0.00421 & 0.00244 & 0.00231 & 0.00230 & \\
\hline$r_{\mathrm{p}}$ & 2 & 0.2634 & & & & 0.0006 \\
\hline$r_{\mathrm{s}} / r_{\mathrm{p}}$ & 3 & 0.891 & & & & 0.002 \\
\hline$r_{\mathrm{s}}$ & & 0.2347 & & & & 0.0002 \\
\hline prim. limb dark. coeff. & 4 & 0.85 & 0.82 & 0.76 & 0.67 & \\
\hline second. limb dark. coeff. & 5 & 0.88 & 0.84 & 0.78 & 0.69 & \\
\hline$i$ & 6 & 87.45 & & & & 0.16 \\
\hline$e \cdot \cos (\omega)$ & 7 & 0.0 & $=$ & $=$ & $=$ & \\
\hline$e \cdot \sin (\omega)$ & 8 & 0.0 & $=$ & $=$ & $=$ & \\
\hline prim. gravita. dark. coeff. & 9 & 0.25 & $=$ & $=$ & $=$ & \\
\hline second. gravita. dark. coeff. & 10 & 0.25 & $=$ & $=$ & $=$ & \\
\hline prim. reflection (internal calculation) & 11 & 0.00851 & 0.00855 & 0.00904 & 0.00937 & \\
\hline second. reflection (internal calculation) & 12 & 0.01435 & 0.01427 & 0.01404 & 0.01373 & \\
\hline masses ratio & 13 & 0.894 & & & & \\
\hline lead/lag ang & 14 & 0.0 & & & & \\
\hline third light & 15 & 0.0 & & & & \\
\hline out of phase & 16 & 0.01165 & 0.01171 & 0.01183 & 0.01175 & \\
\hline maximum light & 17 & -0.01179 & 0.04064 & -0.08383 & -0.18474 & \\
\hline integration ring & 18 & 5 & & & & \\
\hline period & 19 & 0.81687099 & & & & \\
\hline initial epoch- 2440000 & 20 & 3230.609 & & & & \\
\hline$\sigma<(\mathrm{O}-\mathrm{C})>$ & & 0.019571 & 0.011258 & 0.010129 & 0.009652 & \\
\hline
\end{tabular}


Table 6. BH Vir. Absolute parameters

\begin{tabular}{|c|c|c|c|c|c|c|}
\hline Literature & & $\begin{array}{c}P \\
0.81687099 \\
0.00000010 \\
\end{array}$ & $\begin{array}{c}K_{1} \\
138.4 \\
1.0 \\
\end{array}$ & $\begin{array}{c}K_{2} \\
154.9 \\
1.0 \\
\end{array}$ & & \\
\hline Our & EBOP & $\begin{array}{c}J_{\mathrm{S}} \\
0.688 \\
0.002 \\
\end{array}$ & $\begin{array}{c}r_{\mathrm{p}} \\
0.2634 \\
0.0006 \\
\end{array}$ & $\begin{array}{c}r_{\mathrm{s}} \\
0.2348 \\
0.0002 \\
\end{array}$ & $\begin{array}{c}i \\
87.45 \\
0.16 \\
\end{array}$ & \\
\hline $\begin{array}{c}\text { Our } \\
u_{0} \\
11.765\end{array}$ & $\begin{array}{c}\text { photometry } \\
v_{0} \\
10.900\end{array}$ & $\begin{array}{c}b_{0} \\
10.368\end{array}$ & $\begin{array}{c}\text { redd }= \\
y_{0} \\
10.019 \\
0.009\end{array}$ & $\begin{array}{c}0.026 \\
(b-y)_{0} \\
0.348\end{array}$ & $\begin{array}{c}m_{10} \\
0.184\end{array}$ & $\begin{array}{c}c_{10} \\
0.333\end{array}$ \\
\hline Primary & Star & & & & & \\
\hline $\begin{array}{c}A \\
4.744 \\
0.092 \\
\end{array}$ & $\begin{array}{c}m_{\mathrm{p}} \\
1.133 \\
0.017 \\
\end{array}$ & $\begin{array}{c}R_{\mathrm{p}} \\
1.250 \\
0.007 \\
\end{array}$ & $\begin{array}{c}\log \left(g_{\mathrm{p}}\right) \\
4.30 \\
0.01 \\
\end{array}$ & $\begin{array}{c}F_{\mathrm{V}}(p) \\
3.77 \\
0.03 \\
\end{array}$ & $\begin{array}{c}T_{\text {eff }}(p) \\
6158 \\
60 \\
\end{array}$ & \\
\hline $\begin{array}{c}d \\
153 \\
18 \\
\end{array}$ & & $\begin{array}{c}L_{\mathrm{p}} \\
1.98 \\
0.02 \\
\end{array}$ & $\begin{array}{c}M_{\text {bol }}(p) \\
3.90 \\
0.05 \\
\end{array}$ & $\begin{array}{c}B C \\
-0.20 \\
0.27 \\
\end{array}$ & $\begin{array}{c}M_{\mathrm{v}}(p) \\
4.10 \\
0.26 \\
\end{array}$ & \\
\hline \multirow[t]{2}{*}{ Secondary } & $\begin{array}{c}\text { Star } \\
m_{\mathrm{s}} \\
1.013 \\
0.016 \\
\end{array}$ & $\begin{array}{c}R_{\mathrm{S}} \\
1.114 \\
0.006 \\
\end{array}$ & $\begin{array}{c}\log \left(g_{\mathrm{s}}\right) \\
4.35 \\
0.01 \\
\end{array}$ & $\begin{array}{c}F_{\mathrm{v}}(s) \\
3.73 \\
0.03 \\
\end{array}$ & $\begin{array}{c}T_{\text {eff }}(s) \\
5607 \\
60 \\
\end{array}$ & \\
\hline & & $\begin{array}{c}L_{\mathrm{s}} \\
1.08 \\
0.02\end{array}$ & $\begin{array}{c}M_{\text {bol }}(s) \\
4.55 \\
0.05\end{array}$ & $\begin{array}{c}B C \\
-0.20 \\
0.27\end{array}$ & $\begin{array}{c}M_{\mathrm{v}}(s) \\
4.75 \\
0.26\end{array}$ & \\
\hline
\end{tabular}

Etzel P.B., 1981, in: Photometric and Spectroscopic Binary Systems, Carling E.B. and Kopal Z. (eds.) p. 111

Guiricin G., Mardirossian F., Mezzetti M., 1984, MNRAS 206, 305

Hartmann L.W., Noyes R.W., 1987, ARA\&A 25, 271

Hoffmann M., 1982, A\&A 471, 561

Kitamura M., Nakamura T., Takahashi C., 1957, PASP 9, 191

Koch R.H., 1967, AJ 72, 411

Lacy C.N., 1977, ApJ 213, 458

Ludington E.W., 1978, Doctoral Thesis, Florida University

Martinov D.Ya., 1973, in: Eclipsing Variable Stars. John Wiley and Sons, New York, p. 128

Moon T.T., 1985, Ap\&SS 122, 173
Olsen E.H., 1984, A\&AS 57, 443

Olsen E.H., 1988, ApJ 189, 173

Popper D.M., 1995, Inf. Bull. on Variable Stars, No. 4185

Reglero V., Gimenez A., Estela A., 1990, A\&A 231, 375

Sadik A.R., 1978, Doctoral Thesis, U. Manchester

Saxner M., Hammarback G., 1985, A\&A 151, 372

Scaltriti F., Cellino A., Busso M., 1985, A\&A 149, 11

Schmidt-Kaler T., Landolt-Börnstein Eds., Hellwegw K.H., Schaifers K., Voigt HH., 1982. Springer-Verlag, New SE., group VI Vol. 2b, p. 451

Vincent A., Piskunov N.E., Tuominen I., 1993, A\&A 278, 523

Zhai D.S., Qiao G.J., Zhang X.Y., 1990, A\&A 237, 148 